\title{
A novel pair of inducible expression vectors for use in Methylobacterium extorquens
}

\author{
Lon M Chubiz ${ }^{1}$, Jessica Purswani ${ }^{1,2}$, Sean Michael Carroll ${ }^{1}$ and Chistopher J Marx ${ }^{1,3^{*}}$
}

\begin{abstract}
Background: Due to the ever increasing use of diverse microbial taxa in basic research and industrial settings, there is a growing need for genetic tools to alter the physiology of these organisms. In particular, there is a dearth of inducible expression systems available for bacteria outside commonly used $\gamma$-proteobacteria, such as Escherichia coli or Pseudomonas species. To this end, we have sought to develop a pair of inducible expression vectors for use in the $\alpha$-proteobacterium Methylobacterium extorquens, a model methylotroph.
\end{abstract}

Findings: We found that the $P_{R}$ promoter from rhizobial phage 16-3 was active in M. extorquens and engineered the promoter to be inducible by either $p$-isopropyl benzoate (cumate) or anhydrotetracycline. These hybrid promoters, $P_{R / c m t O}$ and $P_{R / t e t O}$, were found to have high levels of expression in $M$. extorquens with a regulatory range of 10 -fold and 30-fold, respectively. Compared to an existing cumate-inducible (10-fold range), high-level expression system for M. extorquens, $P_{R / c m t O}$ and $P_{R / \text { teto }}$ have $33 \%$ of the maximal activity but were able to repress gene expression 3 and 8 -fold greater, respectively. Both promoters were observed to exhibit homogeneous, titratable activation dynamics rather than on-off, switch-like behavior. The utility of these promoters was further demonstrated by complementing loss of function of $\mathrm{ftfL}$ - essential for growth on methanol - where we show $\mathrm{P}_{R / \text { tetO }}$ is capable of not only fully complementing function but also producing a conditional null phenotype. These promoters have been incorporated into a broad-host-range backbone allowing for potential use in a variety of bacterial hosts.

Conclusions: We have developed two novel expression systems for use in M. extorquens. The expression range of these vectors should allow for increased ability to explore cellular physiology in M. extorquens. Further, the $P_{R / t e t O}$ promoter is capable of producing conditional null phenotypes, previously unattainable in M. extorquens. As both expression systems rely on the use of membrane permeable inducers, we suspect these expression vectors will be useful for ectopic gene expression in numerous proteobacteria.

\section{Background}

As the amount of bacterial genome sequencing information continues to grow, the need for broad-host-range, extensible genetic tools will become increasingly ubiquitous. In particular, the capacity for heterologous gene expression in diverse microbial taxa will be of paramount importance for numerous research goals, as well as industrial and synthetic biological applications. To this end, we explored the use of two well-characterized transcriptional repressors (TetR and CymR) in conjunction with

\footnotetext{
*Correspondence: cmarx@oeb.harvard.edu

${ }^{1}$ Department of Organismic and Evolutionary Biology, Harvard University, 16 Divinity Ave., Cambridge, MA 02138, USA

${ }^{3}$ Faculty of Arts and Sciences Center for Systems Biology, Harvard University, Cambridge, MA, USA

Full list of author information is available at the end of the article
}

a phage-derived promoter $\left(\mathrm{P}_{R}\right.$ from phage 16-3) to produce a novel of set inducible expression vectors for use in the facultative methylotroph Methylobacterium extorquens.

Methylotrophic bacteria are a ubiquitous group of microorganisms defined by their capacity to utilize reduced single-carbon $\left(\mathrm{C}_{1}\right)$ compounds as a sole source of energy and biomass. The facultatively methylotrophic, $\alpha$-proteobacterium Methylobacterium extorquens has been a model organism in the study of $\mathrm{C}_{1}$ metabolism for over 50 years. In the last decade, due in part to the development of a repertoire of genetic tools [1-4] Methylobacterium species have become increasingly useful in the study of horizontally transferred metabolic pathways [5-7] and microbial evolution [8-10]. Furthermore, in the past few years genome sequences have become available 
for eight representatives within Methylobacterium [11,12]. While considerable progress has been made for genetic manipulation of $M$. extorquens, an area that remains underrepresented by comparison is the development of regulated expression systems.

To date, only one regulated expression system has been demonstrated to be functional in M. extorquens. Choi and coworkers constructed an inducible expression system utilizing the cumate responsive transcriptional repressor, CymR, from Pseudomonas putida $\mathrm{F} 1$ and the strong $\mathrm{P}_{m x a F}$ promoter that drives the expression of methanol dehydrogenase in $M$. extorquens [13]. This hybrid system has been modified and utilized to test the fitness consequences of gene expression levels of different formaldehyde oxidation enzymes in Methylobacterium [14,15]. While functional, this promoter-operator pairs are extremely "leaky", wherein the basal level of expression in non-inducing conditions is quite high [14]. This limitation makes heterologous gene expression exceedingly difficult, and hampers the exploration of conditionally null phenotypes.

Building on these previous findings, we have employed an additional transcriptional repressor, TetR, from the transposon Tn10. As the foundational member of the TetR-family of DNA binding proteins [16], to whom CymR is also a member, TetR has been extensively studied yielding much data on ligand binding, DNA binding kinetics, and operator site specificity [17]. In the absence of inducer, TetR and CymR bind tightly to their respective operator sites (see Figure 1), thereby inhibiting transcriptional initiation by RNA polymerase. Upon binding of ligands such as tetracycline or anhydrotetracycline (a high-affinity ligand) in the case of TetR, or cumate ( $p$-isopropyl benzoate) with CymR, the affinity of TetR and CymR for their respective operator sites is nearly abolished, allowing for transcription initiation to proceed. Exploiting these characteristics, numerous studies have modified existing expression systems to behave in a dosedependent manner. In fact, TetR and related transcriptional repressors have found use in numerous synthetic biology applications in bacteria, archaea, and eukaryotes [13,18-23].

Here we describe the construction of two IncP-based, inducible expression vectors for use in $M$. extorquens, and possibly numerous other proteobacteria with minor modification. The novelty of these vectors lies in their use of two separate transcriptional repressors, TetR and CymR, along with a strong promoter from the rhizobial phage 16-3. We demonstrate the utility of these vectors by showing that i) induction is dose-dependent, ii) induction is continuous through time, and iii) the regulatory range of both systems exceeds those currently available for $M$. extorquens. Collectively, these results supply researchers investigating $M$. extorquens, and likely numerous other proteobacteria, with two alternative systems to express genes in traditional and synthetic biology applications.

\section{Findings}

Promoter design and rationale

During the process of selecting an appropriate promoter, we desired that the promoter i) be sufficiently active in $M$. extorquens and ii) not be subject to regulation by native transcription factors. Based on these two criteria, a natural source for such a promoter was from bacteriophage. Many bacteriophage promoters have a wide host range and often have strong, constitutive activity in the absence of their transcriptional control mechanisms. However, numerous well characterized coliphage-derived promoters such as $\lambda \mathrm{P}_{L}, \lambda \mathrm{P}_{R}$, T5 $\mathrm{P}_{N 25}$, T7 $\mathrm{P}_{A 1}$ are weakly active or inactive in $M$. extorquens [13]. To this end, we looked to other bacteriophage promoters that have been shown to be active in $\alpha$-proteobacteria. Based on this metric, we explored the use of promoters from the control region of the rhizobial phage $16-3\left(\mathrm{P}_{L}\right.$ and $\left.\mathrm{P}_{R}\right)$. Phage 16-3 has been extensively examined with physiological and biochemical studies in both its host, the $\alpha$-proteobacterium Sinorhizobium meliloti, and Escherichia coli [24,25], suggesting that $\mathrm{P}_{L}$ and $\mathrm{P}_{R}$ may be functional in a variety of hosts. Additionally, the only transcriptional regulator known to interact with $\mathrm{P}_{L}$ and $\mathrm{P}_{R}$ is the $16-3 \mathrm{C}$ repressor [25].

In a set of exploratory experiments, we found that $\mathrm{P}_{R}$ was active in $M$. extorquens (data not shown). As we desired to construct inducible systems, we focused attention to engineering $\mathrm{P}_{R}$ derivatives containing operator sites for the CymR and TetR regulators (Figure 1). The resulting hybrid promoters, $\mathrm{P}_{R / \text { cmtO }}$ and $\mathrm{P}_{R / \text { tetO }}$, were found to produce the widest regulatory range without interfering with $\mathrm{P}_{R}$ promoter activity. Interestingly, we found that placing the operators, specifically tet $O$, throughout other regions of the promoter resulted in either loss of promoter repression or activity (data not shown). This was a somewhat surprising result given the flexibility of many other phage-derived systems to be manipulated with multiple repressor and activator operator sites $[18,26]$. Collectively, these findings allowed us to engineer two inducible promoters with similar maximal activity (Figure 2).

\section{Activation of $\mathrm{P}_{R / \text { cmto }}$ and $\mathrm{P}_{R / \text { teto }}$ is dose-dependent}

A desirable property for regulated expression systems is for levels of gene expression from the promoter to be proportional to the concentration of inducer. In order to explore the range of induction of $\mathrm{P}_{R / c m t O}$ and $\mathrm{P}_{R / t e t O}$, the promoters along with their respective regulatory proteins were introduced onto broad-host-range plasmids (IncP compatibility group) to create the expression vectors pLC290 and pLC291 (Figure 1). Since previous studies 


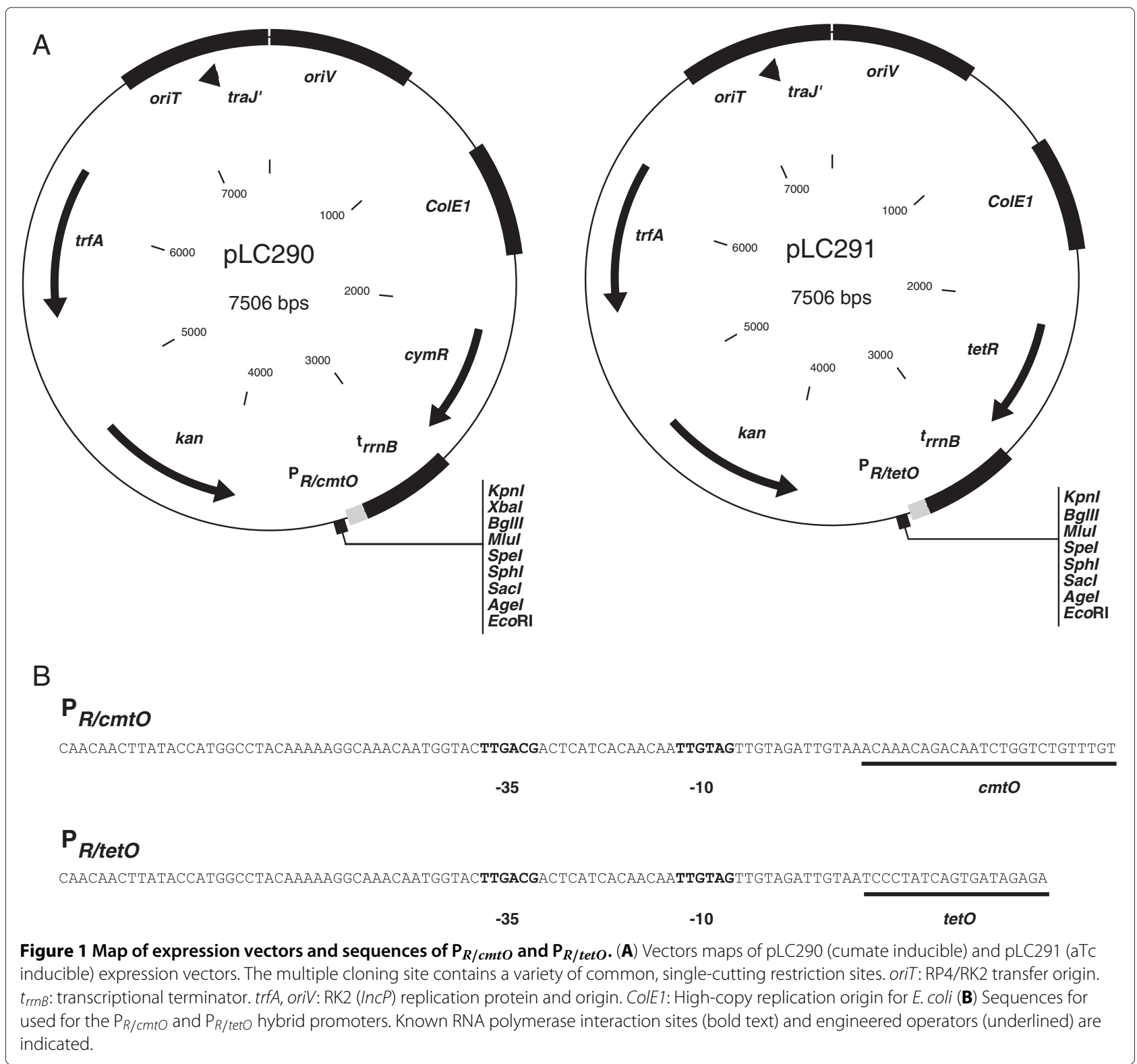

have demonstrated $m$ Cherry to be a sensitive measure of gene expression in $M$. extorquens [14], we decided to use mCherry fluorescence as a metric of promoter activity. We placed the red-fluorescent protein variant $m$ Cherry under the control of each promoter in pLC290 and pLC291 and introduced the resulting vectors (pJP18T and pJP22T) into $M$. extorquens. To induce expression from $\mathrm{P}_{R / c m t O}$ and $\mathrm{P}_{R / t e t O}$, we supplied varied concentrations of cumate (Q) and anhydrotetracycline (aTc), respectively, to $M$. extorquens cultures.

In general, both promoters were found to be responsive to concentrations of $\mathrm{Q}$ and aTc that were in agreement with previous studies in $M$. extorquens or other organisms $[13,18,27]$. The $\mathrm{P}_{R / c m t O}$ promoter was observed to respond to a range of 0.1 to $5 \mu \mathrm{g} / \mathrm{ml}$ ( 0.6 to 30 $\mu \mathrm{M})$ of $\mathrm{Q}$ and the $\mathrm{P}_{R / t e t O}$ promoter from 0.1 to 25 $\mathrm{ng} / \mathrm{ml}(0.2 \mathrm{nM}$ to $50 \mathrm{nM})$ aTc. Interestingly, the induction profile of $\mathrm{P}_{R / c m t O}$ increased in a log-linear fashion over the entire concentration range, whereas $\mathrm{P}_{R / \text { tetO }}$ was observed to have a much more concave profile. In terms of regulatory range, $\mathrm{P}_{R / \text { cmtO } O}$ and $\mathrm{P}_{R / t e t O}$ were observed to have 10-fold and 30-fold induction, respectively, with both promoters having the same maximum absolute levels of expression (Figure 2). Importantly, the basal level of expression from $\mathrm{P}_{R / c m t O}$ was found to be approximately 3-fold higher than that of $\mathrm{P}_{R / t e t O}$. Taken together, these data suggest that while $\mathrm{P}_{R / c m t O}$ may be more tunable, $\mathrm{P}_{R / \text { tetO }}$ serves as a superior expression system for 

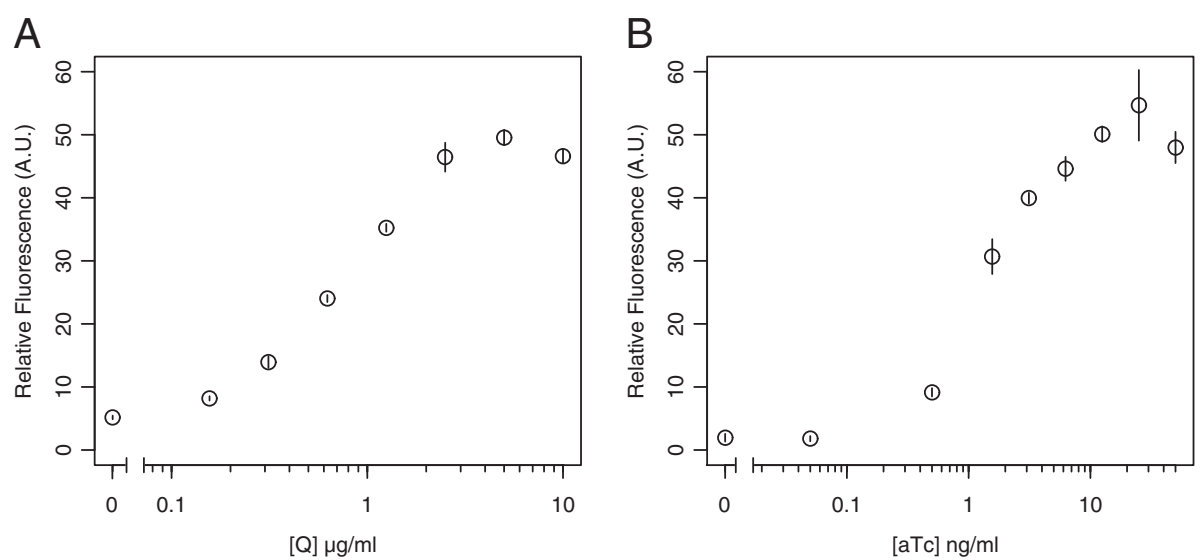

Figure 2 Induction profiles of the $\mathbf{P}_{\boldsymbol{R} / \boldsymbol{c m t} \boldsymbol{O}}$ and $\mathbf{P}_{\boldsymbol{R} / \text { tet } \boldsymbol{O}}$ promoters. Induction profiles of $m$ Cherry containing pLC290 and pLC291 derivatives (A) pJP18T and (B) pJP22T in M. extorquens PA1. Cell cultures were grown to mid-log phase and induced for 24 hrs prior to fluorescence measurements. Fluorescence units are presented as arbitrary units (A.U.) and normalized as described in Materials and Methods.

genes requiring tight repression, such as cytotoxic proteins. Also, we found that there was minimal crosstalk between the CymR and TetR ligand specificity or promoter binding indicating these systems would work independent of one another (pJP18T: 4.6 Uninduced/4.2 with aTc; pJP22T: 1.0 Uninduced/1.1 with Q; Grown in succinate).

Comparing the levels of gene expression and regulatory range of $\mathrm{P}_{R / c m t O}$ and $\mathrm{P}_{R / \text { tetO } O}$ to the cumate inducible $\mathrm{P}_{m x a F}$ promoter previously reported $[13,14]$, we found that in $M$. extorquens these promoters achieve $33 \%$ of the maximal activity of $\mathrm{P}_{m x a F}$ (the strongest known Methylobacterium promoter) and provide a greater degree of repression. Specifically, a cumate-inducible $\mathrm{P}_{m x a F} m$ Cherry expression vector, $\mathrm{pHC} 115 \mathrm{~m}$, yielded relative fluorescence values of $15.6 \pm 1.5$ (uninduced) to $157.1 \pm 3.7$ (induced). While this 10 -fold regulatory range was similar to $\mathrm{P}_{R / c m t O}$, the minimal and maximal expression from $\mathrm{P}_{R / c m t O}$ were both 3-fold lower. By comparison, $\mathrm{P}_{R / \text { tetO }}$, with a 30fold regulatory range, was able to repress expression 8-fold lower than the $\mathrm{P}_{\text {mxaF }}$ system with only a 3-fold difference in maximum expression. Collectively, these results demonstrate that both $\mathrm{P}_{R / c m t O}$ and $\mathrm{P}_{R / \text { tetO }}$ provide improvement over previously explored systems. However, we do note that $\mathrm{P}_{m x a F}$ may remain a superior promoter in cases when high-level protein over-expression is desired.
Importantly, these hybrid promoters allow for more relevant exploration of cellular physiology as their expression levels and ranges fall well within or above native promoters in $M$. extorquens.

\section{Maximal activation of $\mathrm{P}_{R / c m t O}$ and $\mathrm{P}_{R / \text { teto }}$ is substrate dependent}

An issue with many expression systems designed with host-derived promoters is the possibility of interactions with native transcription factors. Specifically, the $\mathrm{P}_{m x a F}$ promoter is known to be more highly active in cells grown on methanol as opposed to succinate $[1,28]$. To explore this possibility, with respect to $\mathrm{P}_{R / c m t O}$ and $\mathrm{P}_{R / \text { tetO }}$, we cultured $M$. extorquens harboring $\mathrm{pJP} 18 \mathrm{~T}$ and pJP22T in media with either methanol or succinate as the sole carbon source (Table 1). We found that succinate grown cells possessed a nearly 2 -fold increase in maximal gene expression, compared to methanol grown cells; effectively, the opposite behavior seen with $\mathrm{P}_{\text {mxaF }}$. We suspect that this disparity in maximal expression may be due to an external factor, such as different plasmid copy numbers, between methanol and succinate growth. Previously reported XylE and $\beta$-galactosidase promoter probe vectors used in $M$. extorquens, such as pCM130 and pCM132 (plasmids with the same backbone as pLC290 and pLC291), exhibit between 2 and

Table 1 Growth substrate dependence on $\mathrm{P}_{R / \text { cmtO }}$ and $\mathrm{P}_{R / \text { tetO }}$ activation

\begin{tabular}{|c|c|c|c|c|c|}
\hline \multirow[b]{2}{*}{ Plasmid } & \multirow[b]{2}{*}{ Promoter } & \multicolumn{2}{|c|}{ Methanol } & \multicolumn{2}{|c|}{ Succinate } \\
\hline & & Uninduced & Induced & Uninduced & Induced \\
\hline pJP18T & $\mathrm{P}_{\mathrm{R} / \mathrm{cm} t \mathrm{O}}$ & $5.32 \pm 0.64$ & $28.94 \pm 2.14$ & $5.79 \pm 0.44$ & $61.93 \pm 2.42$ \\
\hline pJP22T & $\mathrm{P}_{\text {R/tetO }}$ & $1.46 \pm 0.27$ & $33.13 \pm 2.63$ & $1.95 \pm 0.62$ & $54.67 \pm 5.60$ \\
\hline
\end{tabular}

Gene expression as measured by $m$ Cherry fluorescence from $M$. extorquens cells harboring pJP18T or pJP22T. Cells were grown in succinate or methanol medium in the presence or absence of Q $(5 \mu \mathrm{g} / \mathrm{ml})$ or aTc $(25 \mathrm{ng} / \mathrm{ml})$. Values are relative fluorescence (arbitrary units) and reported error is the $95 \%$ confidence interval ( $\mathrm{N}=4)$. 
3-fold increases in background activity during succinate versus methanol growth [1]. As pCM130 and pCM132 possess no promoter sequences upstream of their reporter genes, the only likely variation that might exist is in plasmid copy number. Comparing these findings to our own, where $\mathrm{P}_{R / \text { cmtO }}$ and $\mathrm{P}_{R / \text { tetO }}$ contain no host-related transcription factor binding sites, we see similar fold changes in maximal expression suggesting that a similar mechanism may be affecting these expression systems. Taken together, these data indicate that single-copy or chromosomally integrated systems be used in situations where uniform expression is desired across substrates.

\section{Induction of $\mathrm{P}_{R / \text { cmto }}$ and $\mathrm{P}_{R / \text { teto }}$ is continuous}

A problematic feature of many expression systems, particularly those associated with metabolic pathways, is that gene expression can exhibit phenotypic heterogeneity throughout the population of cells, such as an on-off, switch-like behavior [29-31]. To explore this possibility, we grew $M$. extorquens strains bearing the $m$ Cherry expression vectors $\mathrm{pJP} 18 \mathrm{~T}$ and $\mathrm{pJP} 22 \mathrm{~T}$ to mid$\log$ phase, induced cultures with either $\mathrm{Q}$ or aTc, and measured the time course of individual-cell fluorescence by flow cytometry. We found that over 8 hours of induction the induced populations activated transcription in a uniform, continuous manner (Figure 3). Though we did observe residual uninduced cells, we suspect this may be due to debris introduced by our cell fixing method or possibly cells losing $m$ Cherry due to costly over-expression. These data demonstrate the utility of the $\mathrm{P}_{R / c m t O}$ and $\mathrm{P}_{R / \text { tetO }}$ expression systems in studying aspects of cellular physiology requiring uniform gene expression.

\section{Complementation and conditional null phenotypes using $\mathrm{P}_{R / \text { teto }}$ constructs}

To examine the utility of these vectors for studying $M$. extorquens physiology, we complemented a gene encoding a key enzyme in methanol metabolism using the $\mathrm{P}_{R / \text { tetO- }}$ based plasmid pLC291. We chose to use utilize $\mathrm{P}_{R / \text { tetO }}$ due to the tight induction properties we have observed using an mCherry reporter (Figure 2 and Table 1). The product of $f t f L$ (formate-tetrahydrofolate ligase) is required for the assimilation of formate into biomass during one-carbon metabolism [32]. A disruption in $f t f L$ results in a methanol minus growth phenotype. By complementing a $f t f L$ knockouts using $f t f L$-expressing vectors under the control of $\mathrm{P}_{R / \text { tetO }}$, in the presence of aTc, we found that we could fully restore growth on methanol (Figure 4). Importantly, in the absence of aTc, we observed that we were able to produce a complete null phenotype for $f t f L$ (Figure 4). To date, no expression system for $M$. extorquens has been capable of producing conditional null phenotypes. These results demonstrate the utility of $\mathrm{P}_{R / \text { tet } O}$ to study $\mathrm{M}$. extorquens physiology and generate conditional null mutants regulated by aTc.

\section{Conclusions}

To date, only a handful of expression systems exist for bacterial models outside $E$. coli and other closely related $\gamma$-proteobacteria. In an effort to expand the genetic toolkit available to researchers working with $M$. extorquens, and presumably other proteobacteria, we have constructed a set of two inducible expression vectors that utilize the CymR and TetR (cumate and tetracycline repressors) in conjunction with the strong $\mathrm{P}_{R}$ promoter from phage 16-3. The pLC290 and pLC291 vectors were found to provide uniform, high-level expression in $M$.
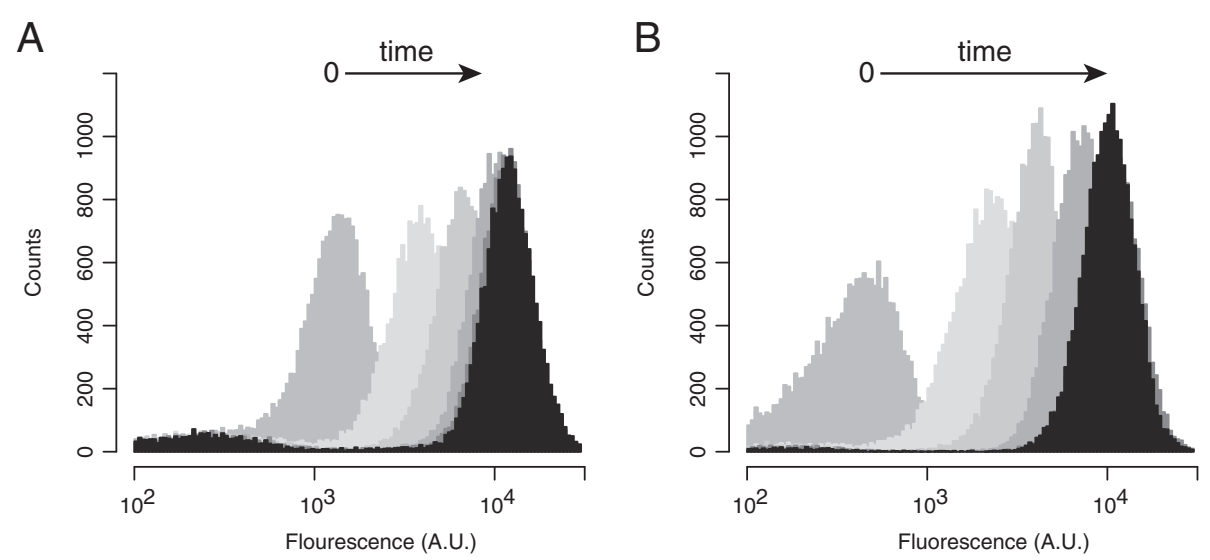

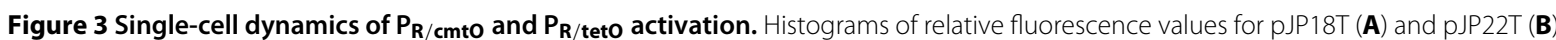
harboring M. extorquens PA1 as determined by single-cell flow cytometry. Cultures were grown to mid-log phase and induced with $5 \mu \mathrm{g} / \mathrm{ml} \mathrm{Q}(\mathbf{A})$ or $25 \mathrm{ng} / \mathrm{ml}$ aTc (B). At times 0, 2, 4, 6, 8, and $24 \mathrm{hrs}$, cells were harvested and fixed in carbon-free Hypho medium supplemented with $100 \mathrm{mg} / \mathrm{ml}$ streptomycin. The 8 and $24 \mathrm{hr}$ time points have nearly overlapping fluorescence distributions. Fluorescence units are presented as arbitrary units (A.U.) and normalized as described in Materials and Methods. 


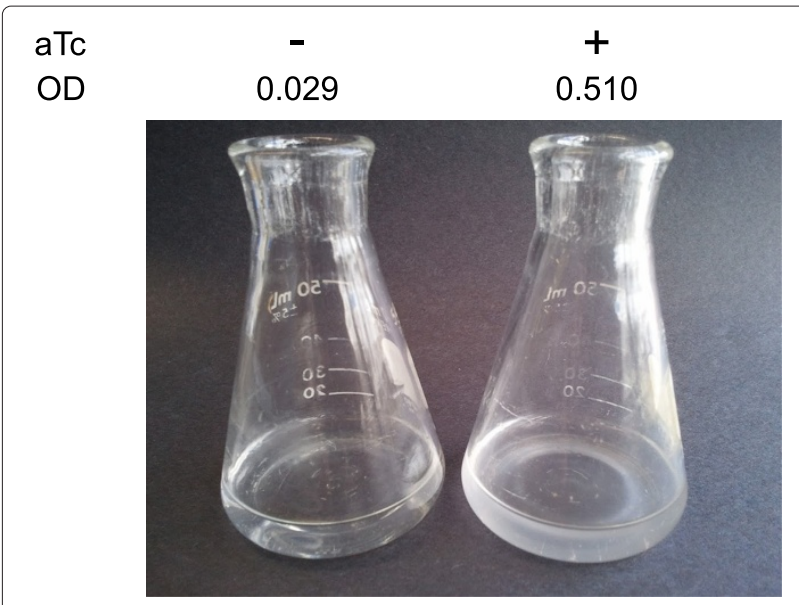

Figure $\mathbf{4}$ Complementation and conditional null phenotype of $\mathrm{ftfL}$. Images of methanol grown cultures of $M$. extorquens AM1 strains CM4103 in the presence (+) and absence (-) of $20 \mu \mathrm{g} / \mathrm{ml}$ aTc. Final $\mathrm{OD}(600 \mathrm{~nm})$ values presented are after $72 \mathrm{hrs}$ of growth at $30^{\circ} \mathrm{C}$ in 20 $\mathrm{mM}$ MeOH supplemented medium.

extorquens over a wide range of inducer concentrations. Importantly, compared to the only existing inducible system for $M$. extorquens, we found that $\mathrm{P}_{R / c m t O}$ and $\mathrm{P}_{R / \text { tetO }}$ have 3 and 8 -fold increases in repression, respectively. This provides a significant improvement in the ability to explore $M$. extorquens cellular physiology. Further, as these promoters operate orthogonally to one another, we believe these expression systems will easily work in concert within a single strain to allow complex genetic engineering in a wider range of bacteria. For these reasons, we believe these vectors and promoter systems will be of great use to the bacteriological community in many research and industrial settings.

\section{Availability of supporting information}

The plasmid data supporting the results of this article are available in the AddGene repository with identification numbers http://www.addgene.org/44447/ and http:// www.addgene.org/44448/.

\section{Methods}

\section{Bacterial strains, medium, and growth conditions}

All bacterial strains used in this work are derivatives of Escherichia coli NEB10 $\beta$ (New England Biolabs), E.

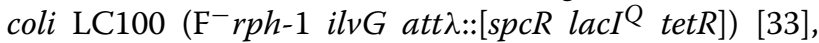
Methylobacterium extorquens PA1 strain CM2730 ( $\Delta$ celABCD) [34] or $M$. extorquens AM1. Growth of all strains, except E. coli, was performed in modified 'Hypho' minimal medium as described by Chou and coworkers [10], with succinate at $5 \mathrm{mM}$ or methanol at $20 \mathrm{mM}$. $E$. coli strains were cultured in Luria-Bertani broth as described by Miller [35] or nutrient broth. Media was supplemented with kanamycin at $50 \mu \mathrm{g} / \mathrm{ml}$ or ampicillin at $100 \mu \mathrm{g} / \mathrm{ml}$ to select for the presence of all plasmids. Inducers anhydrotetracycline (aTc) and cumate- $\mathrm{KOH}(\mathrm{Q})$ were supplied at $25 \mathrm{ng} / \mathrm{ml}$ or $5 \mu \mathrm{g} / \mathrm{ml}$ from aqueous stocks, respectively, unless otherwise indicated. Growth and gene expression experiments were performed at $30^{\circ} \mathrm{C}$ using an automated growth system described by Delaney and coworkers $[34,36]$.

\section{Plasmid and strain construction}

Promoter designs were initially constructed and subsequently mutated in a pBluescript(SK-) (Stratagene) backbone. Synthetic oligonucleotides CAACAACTTATACC ATGGCCTACAAAAAGGCAAACAATGGTACTTGAC GACTCATCACAA and GTCCGTTCGTTACAATCTA CAACTACAATTGTTGTGATGAGTCGTCAAGTACC ATTG containing the sequence for a 91 nt region encoding the $\mathrm{P}_{R}$ promoter from the rhizobial phage 16-3. The oligonucleotides were annealed to form a $91 \mathrm{bp}$ dsDNA fragment, followed by PCR amplification with primers ATAGGGCCCCAACAACTTATACCATGGCC TAC and ATAGGTACCGTCCGTTCGTTACAATCTA CAAC to introduce PspOMI and KpnI restriction sites. The resulting fragment was digested with PspOMI and $K p n I$ and cloned into the respective sites in pBluescript(SK-) to form pLC265. TetR and CymR operator sites (tet $O$ and $c m t O)$, were introduced at the distal end of $\mathrm{P}_{R}$ in pLC265 using enzymatic inverse PCR (EI-PCR) [37] using primers ATACGTCTCATCCCTATCAGTGA TAGAGAGTTGTAGATTGTAACGAACGGAC, ATAC GTCTCAGGGACGTCAAGTACCATTGTTTGCC, AT ACGTCTCAACAAACAGACAATCTGGTCTGTTTGT GGTACCCAATTCGCCCTATAG, and ATACGTCTCA TTGTTTACAATCTACAACTACAATTGTTGTG followed by $B s m B I$ digestion and ligation to generate plasmids pLC271 ( $\mathrm{P}_{R / t e t O}$ containing) and pLC277 $\left(\mathrm{P}_{R / \text { cmtO }}\right.$ containing).

The subsequent broad-host-range vectors were constructed using the expression vector pHC115 [14] as a template. A DNA region encoding Tn10 tet $R$ was PCR amplified from LC100 using primers ATAGCT AGCAGGGAGAGACCCCGAATGATGTCTAGATTAG ATAAAAGTAAAGTG and ATAGGGCCCTTAAGACC CACTTTCACATTTAAG containing NheI and PspOMI restriction sites. The resulting product was digested and ligated into the NheI and PspOMI sites of pHC115, thereby replacing the $c y m R$ coding region with tetR to form pLC261. From $\mathrm{pHC} 115$ and pLC261, the $\mathrm{P}_{m x a F}$ region was excised with $P s p \mathrm{OMI}$ and $K p n \mathrm{I}$ and replaced with subcloned $\mathrm{P}_{R / c m t O}$ and $\mathrm{P}_{R / \text { tetO }}$ fragments from pLC277 and pLC271. To the resulting plasmids, a $\mathrm{t}_{r r n B}$ terminator was PCR amplified from pHC01 [10] using primers ACGCGAAATTCAAGCGC TAGGGCCAAGTTGGGTAACGCCAGGGTTTTCCC or ATGTGAAAGTGGGTCTTAAGGGCCAAGTTGG 
GTAACGCCAGGGTTTTCCC and TGTAGGCCAT GGTATAAGTTGTTGGGATGCAAAAACGAGGCTAG TTTACC and cloned into the PspOMI site, using the method of Gibson and coworkers [38], to reduce transcriptional read-through into the $\mathrm{P}_{R / \text { cmtO }}$ and $\mathrm{P}_{R / \text { tetO }}$ promoter regions. Likewise a more comprehensive multiple cloning site was introduced into the $K p n \mathrm{I}$ and $E c o$ RI sites using annealed synthetic oligonucleotides GATAG GTACCTCTAGAAGATCTACGCGTACTAGTGCATG CGAGCTCACCGGTGAATTCATAG and CTATGAAT TCACCGGTGAGCTCGCATGCACTAGTACGCGTAG ATCTTCTAGAGGTACCTATC to produce the final expression vectors pLC290 and pLC291. The $m$ Cherry expression vectors $\mathrm{pJP} 18 \mathrm{~T}$ and $\mathrm{pJP} 22 \mathrm{~T}$ were created by subcloning a $K p n \mathrm{I}$ and $E c o$ RI digestion product containing $m$ Cherry from pHC115m [14] into the corresponding sites in pLC290 and pLC291, respectively. The vectors pLC290 (GenBank Accession KC296704) and pLC291 (GenBank Accession KC296705) are publically available from the non-profit organization AddGene.org (http:// www.addgene.org/Christopher_Marx).

Unmarked ftfL knockouts were generated by transforming the the Cre-recombinase expression plasmid pCM157 [3] into $M$. extorquens AM1 derivatives CM216K.1 [39] generating strain CM2336 ( $\Delta f t f L:: l o x P)$. The $f t f L$ omplementation vector was generated by subcloning a KpnI and EcoRI digestion product of a pHC115-based $f t f L$ plasmid (SMC unpublished) into the corresponding sites of pLC291, creating plasmids pSC54. The vector, pSC54, was introduced into CM2336 via triparental mating using the helper plasmid pRK2073 [40,41], to produce strains CM4103 ( $\triangle f t f L$ :: loxP/pSC54). Complementation was performed by inoculation of succinate grown CM4103 into methanol minimal medium containing $0 \mu \mathrm{g} / \mathrm{ml}$ or $20 \mu \mathrm{g} / \mathrm{ml}$ aTc.

\section{Fluorescence-based expression assays}

Assays to measure levels of mCherry protein expression were performed as follows. For dose-dependent response curves, $M$. extorquens strains harboring PJP18T or pJP22T were grown to saturation in $10 \mathrm{ml}$ of Hypho-succinate medium. These cultures were then diluted 1:200 in fresh medium, followed by $630 \mu \mathrm{l}$ aliquots being dispensed to clear, flat-bottom, 48-well microtiter plates (Costar). Cultures were grown for $4 \mathrm{hrs}$ on a plate shaking tower (Caliper) at $150 \mathrm{rpm}$ in a $30^{\circ} \mathrm{C}$ humidified room. After $4 \mathrm{hrs}$ of growth, $10 \mu \mathrm{l}$ of fresh medium containing Q or aTc was added to supply Q and aTc at desired concentrations. Cultures were allowed to continue growth for an additional $24 \mathrm{hrs}$ prior to fluorescence (excitation $587 \mathrm{~nm} / \mathrm{emission} 610 \mathrm{~nm}$ ) and optical density $(600 \mathrm{~nm})$ measurements made using a Tecan Safire2 plate reader. Relative fluorescence values reported are:

$$
\text { Relative fluorescence }(\text { A.U. })=\frac{R F U}{O D_{600}} * 10^{-3}
$$

Dynamic expression assays were conducted under similar conditions as above with the following exceptions. Cells $(200 \mu \mathrm{l}$ of culture) were harvested after induction at $0,2,4,6,8$, and $24 \mathrm{hrs}$. Culture samples were pelleted by centrifugation $(6,000 \mathrm{xg})$ and resuspended in an equal volume of cold Hypho medium without succinate and supplemented with $100 \mathrm{mg} / \mathrm{ml}$ streptomycin to inhibit $m$ Cherry translation. Fixed cells were kept on ice prior to fluorescence measurements made using a BD LSR II Flow Cytometer. Flow cytometry data were then analyzed using the BioConductor flowCore package in R [42]. Reported fluorescence values for flow cytometry are raw values from the BD LSR II and were not correlated to those of the Tecan Safire2.

\section{Competing interests \\ The authors (LMC, JP, SMC and CJM) declare no competing interests with respect to the findings in this article.}

\section{Authors' contributions}

LMC and CJM were responsible for the conception and design of the study. $L M C, J P$, and SMC constructed all vectors and conducted all growth and fluorescence measurement experiments. LMC, SMC, and CJM drafted the manuscript. All authors read and approved the final manuscript.

\section{Acknowledgements}

The authors would like to thank Dipti Nayak for testing preliminary versions of the vectors and Joshua Michener for helpful comments during the drafting of the manuscript. This work was supported by a grant from NIH (GM078209).

\section{Author details}

${ }^{1}$ Department of Organismic and Evolutionary Biology, Harvard University, 16 Divinity Ave., Cambridge, MA 02138, USA. ²Environmental Microbiology Group, Institute of Water Research, University of Granada, C/Ramón y Cajal no. 4, 18071 Granada, Spain. ${ }^{3}$ Faculty of Arts and Sciences Center for Systems Biology, Harvard University, Cambridge, MA, USA.

Received: 11 December 2012 Accepted: 27 March 2013 Published: 6 May 2013

\section{References}

1. Marx CJ, Lidstrom ME: Development of improved versatile broad-host-range vectors for use in methylotrophs and other Gram-negative bacteria. Microbiology 2001, 147(Pt 8):2065-2075.

2. Marx CJ, Lidstrom ME: Development of an insertional expression vector system for Methylobacterium extorquens AM1 and generation of null mutants lacking $m t d A$ and/or fch. Microbiology 2004, 150(Pt 1):9-19.

3. Marx CJ, Lidstrom ME: Broad-host-range cre-lox system for antibiotic marker recycling in gram-negative bacteria. Biotechniques 2002, 33(5):1062-1067.

4. Marx CJ: Development of a broad-host-range sacB-based vector for unmarked allelic exchange. BMC Res Notes 2008, 1:1. [http://dx.doi.org/ 10.1186/1756-0500-1-1]

5. Chistoserdova L, Vorholt JA, Thauer RK, Lidstrom ME: C1 transfer enzymes and coenzymes linking methylotrophic bacteria and methanogenic Archaea. Science 1998, 281(5373):99-102.

6. Chistoserdova L, Jenkins C, Kalyuzhnaya MG, Marx CJ, Lapidus A, Vorholt JA, Staley JT, Lidstrom ME: The enigmatic planctomycetes may hold a key to the origins of methanogenesis and methylotrophy. Mol Biol Evol 2004, 21(7):1234-1241. [http://dx.doi.org/10.1093/molbev/msh113]

7. Kalyuzhnaya MG, Korotkova N, Crowther G, Marx CJ, Lidstrom ME, Chistoserdova L: Analysis of gene islands involved in 
methanopterin-linked $\mathrm{C} 1$ transfer reactions reveals new functions and provides evolutionary insights. $J$ Bacterio/ 2005,

187(13):4607-4614. [http://dx.doi.org/10.1128/JB.187.13.4607-4614.2005]

8. Lee MC, Chou HH, Marx CJ: Asymmetric, bimodal trade-offs during adaptation of Methylobacterium to distinct growth substrates. Evolution 2009, 63(11):2816-2830. [http://dx.doi.org/10.1111/j.1558-5646. 2009.00757.x]

9. Chou HH, Chiu HC, Delaney NF, Segrè D, Marx CJ: Diminishing returns epistasis among beneficial mutations decelerates adaptation. Science 2011, 332(6034):1190-1192. [http://dx.doi.org/10.1126/science. 1203799]

10. Chou HH, Berthet J, Marx CJ: Fast growth increases the selective advantage of a mutation arising recurrently during evolution under metal limitation. PLoS Genet 2009, 5(9):e1000652. [http://dx.doi.org/10. 1371/journal.pgen.1000652

11. Vuilleumier S, Chistoserdova L, Lee MC, Bringel F, Lajus A, Zhou Y, Gourion B, Barbe V, Chang J, Cruveiller S, Dossat C, Gillett W, Gruffaz C, Haugen E, Hourcade E, Levy R, Mangenot S, Muller E, Nadalig T, Pagni M, Penny C, Peyraud R, Robinson DG, Roche D, Rouy Z, Saenampechek C, Salvignol G, Vallenet D, Wu Z, Marx CJ, Vorholt JA, Olson MV, Kaul R, Weissenbach J, Médigue C, Lidstrom ME: Methylobacterium genome sequences: a reference blueprint to investigate microbial metabolism of C1 compounds from natural and industrial sources. PLoS One 2009, 4(5):e5584. [http://dx.doi.org/10.1371/journal.pone.0005584]

12. Marx CJ, Bringel F, Chistoserdova L, Moulin L, Farhan UI Haque M, Fleischman DE, Gruffaz C, Jourand P, Knief C, Lee MC, Muller EEL, Nadalig T, Peyraud R, Roselli S, Russ L, Goodwin LA, Ivanova N, Kyrpides N, Lajus A Land ML, Médigue C, Mikhailova N, Nolan M, Woyke T, Stolyar S, Vorholt JA, Vuilleumier S: Complete genome sequences of six strains of the genus Methylobacterium. J Bacterio/ 2012, 194(17):4746-4748. [http:// dx.doi.org/10.1128/JB.01009-12]

13. Choi YJ, Morel L, Bourque D, Mullick A, Massie B, Míguez CB: Bestowing inducibility on the cloned methanol dehydrogenase promoter (PmxaF) of Methylobacterium extorquens by applying regulatory elements of Pseudomonas putida F1. Appl Environ Microbiol 2006, 72(12):7723-7729. [http://dx.doi.org/10.1128/AEM.02002-06]

14. Chou HH, Marx CJ: Optimization of gene expression through divergent mutational paths. Cell Rep 2012, 1(2):133-140. [http://dx.doi. org/10.1016/j.celrep.2011.12.003]

15. Agashe D, Martinez-Gomez NC, Drummond D A, Marx CJ: Good codons, bad transcript: large reductions in gene expression and fitness arising from synonymous variants in a key enzyme. Mol Biol Evol 2012. in press.

16. Ramos JL, Martínez-Bueno M, Molina-Henares AJ, Terán W, Watanabe K, Zhang X, Gallegos MT, Brennan R, Tobes R: The TetR family of transcriptional repressors. Microbiol Mol Biol Rev 2005, 69(2):326-356. [http://dx.doi.org/10.1128/MMBR.69.2.326-356.2005]

17. Hillen W, Berens $C$ : Mechanisms underlying expression of Tn 10 encoded tetracycline resistance. Annu Rev Microbio/ 1994, 48:345-369. [http://dx.doi.org/10.1146/annurev.mi.48.100194.002021]

18. Lutz R, Bujard $\mathrm{H}$ : Independent and tight regulation of transcriptional units in Escherichia coli via the LacR/O, the TetR/O and AraC/I1-12 regulatory elements. Nucleic Acids Res 1997, 25(6):1203-1210.

19. Wu K, Rao CV: The role of configuration and coupling in autoregulatory gene circuits. Mol Microbio/ 2010, 75(2):513-527. [http://dx.doi.org/10.1111/j.1365-2958.2009.07011.x]

20. Mullick A, Xu Y, Warren R, Koutroumanis M, Guilbault C, Broussau S, Malenfant F, Bourget L, Lamoureux L, Lo R, Caron AW, Pilotte A, Massie B: The cumate gene-switch: a system for regulated expression in mammalian cells. BMC Biotechnol 2006, 6:43. [http://dx.doi.org/10.1186/ 1472-6750-6-43]

21. Gossen $\mathrm{M}$, Bujard $\mathrm{H}$ : Tight control of gene expression in mammalian cells by tetracycline-responsive promoters. Proc Natl Acad Sci USA 1992, 89(12):5547-5551.

22. Gossen M, Freundlieb S, Bender G, Müller G, Hillen W, Bujard H: Transcriptional activation by tetracyclines in mammalian cells. Science 1995, 268(5218):1766-1769.

23. Guss AM, Rother M, Zhang JK, Kulkarni G, Metcalf WW: New methods for tightly regulated gene expression and highly efficient chromosomal integration of cloned genes for Methanosarcina species. Archaea 2008, 2(3):193-203.
24. Dallmann G, Papp P, Orosz L: Related repressor specificity of unrelated phages. Nature 1987, 300:398-401.

25. Papp PP, Nagy T, Ferenczi S, Elõ P, Csiszovszki Z, Buzás Z, Patthy A, Orosz L: Binding sites of different geometries for the 16-3 phage repressor. Proc Natl Acad Sci U S A 2002, 99(13):8790-8795. [http://dx.doi.org/10. 1073/pnas.132275399]

26. Lanzer $\mathrm{M}, \mathrm{Bujard} \mathrm{H}$ : Promoters largely determine the efficiency of repressor action. Proc Natl Acad Sci U S A 1988, 85(23):8973-8977.

27. Choi YJ, Morel L, François TL, Bourque D, Bourget L, Groleau D, Massie B, Míguez CB: Novel, versatile, and tightly regulated expression system for Escherichia coli strains. Appl Environ Microbiol 2010 76(15):5058-5066. [http://dx.doi.org/10.1128/AEM.00413-10]

28. Zhang M, FitzGerald KA, Lidstrom ME: Identification of an upstream regulatory sequence that mediates the transcription of mox genes in Methylobacterium extorquens AM1. Microbiology 2005, 151 (Pt 11):3723-3728. [http://dx.doi.org/10.1099/mic.0.28243-0]

29. Siegele DA, Hu JC: Gene expression from plasmids containing the araBAD promoter at subsaturating inducer concentrations represents mixed populations. Proc Natl Acad SciU S A 1997 94(15):8168-8172.

30. Ozbudak EM, Thattai M, Lim HN, Shraiman BI, Van Oudenaarden A: Multistability in the lactose utilization network of Escherichia coli. Nature 2004, 427(6976):737-740. [http://dx.doi.org/10.1038/nature02298]

31. Novick A, Weiner M: Enzyme induction as an all-or-none phenomenon. Proc Natl Acad Sci U S A 1957, 43(7):553-566.

32. Marx CJ, O'Brien BN, Breezee J, Lidstrom ME: Novel methylotrophy genes of Methylobacterium extorquens AM1 identified by using transposon mutagenesis including a putative dihydromethanopterin reductase. J Bacterio/ 2003, 185(2):669-673.

33. Chubiz LM, Rao CV: Computational design of orthogonal ribosomes. Nucleic Acids Res 2008, 36(12):4038-4046. [http://dx.doi.org/10.1093/nar/ gkn354]

34. Delaney NF, Kaczmarek ME, Ward LM, Swanson PK, Lee MC, Marx CJ: Development of an optimized medium, strain and high-throughput culturing methods for Methylobacterium extorquens. PLOS One 2012, 8(4):e62957.

35. Miller JH: A Short Course in Bacterial Genetics: A Laboratory Manual and Handbook for Escherichia coli and Related Bacteria. Cold Spring Harbor: Cold Spring Harbor Laboratory Press; 1992

36. Delaney NF, Echenique JIR, Marx CJ: Clarity: An open-source manager for laboratory automation. J Lab Autom 2012. [http://dx.doi.org/10. 1177/2211068212460237]

37. Stemmer WP, Morris SK: Enzymatic inverse PCR: a restriction site independent, single-fragment method for high-efficiency, site-directed mutagenesis. Biotechniques 1992, 13(2):214-220

38. Gibson DG, Young L, Chuang RY, Venter JC, Hutchison CA 3rd, Smith HO Enzymatic assembly of DNA molecules up to several hundred kilobases. Nat Methods 2009, 6(5):343-345. [http://dx.doi.org/10.1038/ nmeth.1318]

39. Marx CJ, Laukel M, Vorholt JA, Lidstrom ME: Purification of the formate-tetrahydrofolate ligase from Methylobacterium extorquens AM1 and demonstration of its requirement for methylotrophic growth. J Bacterio/ 2003, 185(24):7169-7175.

40. Chistoserdov AY, Chistoserdova LV, McIntire WS, Lidstrom ME: Genetic organization of the mau gene cluster in Methylobacterium extorquens AM1: complete nucleotide sequence and generation and characteristics of mau mutants. J Bacteriol 1994, 176(13):4052-4065.

41. Figurski DH, Helinski DR: Replication of an origin-containing derivative of plasmid RK2 dependent on a plasmid function provided in trans. Proc Natl Acad Sci USA 1979, 76(4):1648-1652.

42. Hahne F, LeMeur N, Brinkman RR, Ellis B, Haaland P, Sarkar D, Spidlen J, Strain E, Gentleman R: flowCore: a Bioconductor package for high throughput flow cytometry. BMC Bioinformatics 2009, 10:106. [http:// dx.doi.org/10.1186/1471-2105-10-106]

doi:10.1186/1756-0500-6-183

Cite this article as: Chubiz et al:: A novel pair of inducible expression vectors for use in Methylobacterium extorquens. BMC Research Notes 2013 6:183. 